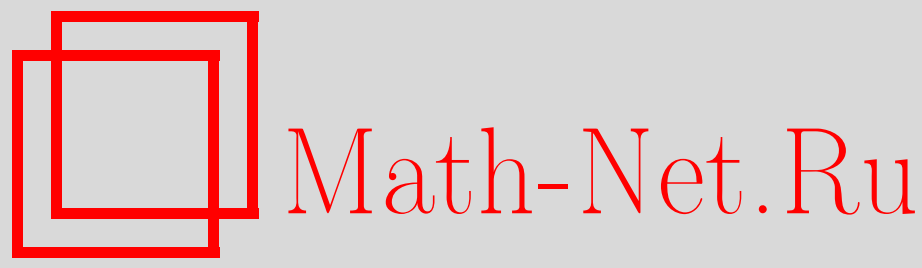

Н. Р. Садыков, Воздействие мощных наносекундных электрических импульсов на массив двухслойных нанотрубок, ТМФ, 2013, том 177, номер 1, 163-176

DOI: https://doi.org/10.4213/tmf8524

Использование Общероссийского математического портала Math-Net.Ru подразумевает, что вы прочитали и согласны с пользовательским соглашением http://www.mathnet.ru/rus/agreement

Параметры загрузки:

IP : 3.81 .55 .215

26 апреля 2023 г., 14:29:06

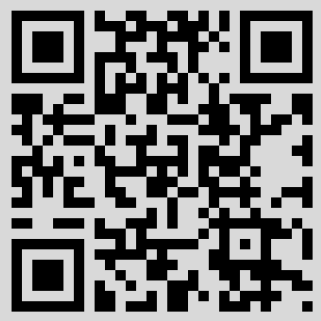




\title{
ФИЗИКА
}

Том 177, № 1

октябрь, 2013

(C) 2013 г.

Н. Р. Садыков*

\section{ВОЗДЕЙСТВИЕ МОЩНЫХ НАНОСЕКУНДНЫХ ЭЛЕКТРИЧЕСКИХ ИМПУЛЬСОВ НА МАССИВ ДВУХСЛОЙНЫХ НАНОТРУБОК}

\begin{abstract}
Получена система уравнений для поверхностной плотности тока с учетом эффекта межоболочечного туннелирования в массиве двухслойных углеродных нанотрубок при наличии нестационарного электрического импульса. Показано, что в отсутствие туннелирования, кроме увеличения их концентрации в два раза в поперечном относительно оси нанотрубок направлении, происходит увеличение резонансной частоты поверхностных токов для симметричной моды примерно в $\sqrt{2}$ раз по сравнению с однослойными нанотрубками. Показано, что изгиб поверхностей углеродных нанотрубок не влияет на величину электропроводности в миллиметровом диапазоне генерируемого излучения. Обсуждается влияние нестационарного электрического импульса на асимметричную моду продольных поверхностных токов.
\end{abstract}

Ключевые слова: двухслойная нанотрубка, массив нанотрубок, взаимодействия излучения с нанотрубками, короткие электрические импульсы.

DOI: $10.4213 / \operatorname{tmf} 8524$

\section{1. ВВЕДЕНИЕ}

В последнее время внимание исследователей привлекают низкоразмерные структуры (наноструктуры): фуллерены, однослойные и многослойные нанотрубки (НТ), нанопроволочки, наноструктуры на основе молекулы ДНК и т. п. В зависимости от радиуса и схемы сворачивания графитовой плоскости НТ могут быть проводниками, полуметаллами и полупроводниками. Зависимость наводимого тока от приложенного напряжения определяет характеристики НТ как электрического проводника [1]-[10]. Проводимость НТ определяется не классическими, а квантовыми законами движения $\pi$-электронов. Процесс переноса заряда под действием приложенного напряжения осуществляется по четырем каналам: двукратному спиновому вырождению и двукратному вырождению зонной структуры в точке уровня Ферми. Если

*Национальный исследовательский ядерный институт "МИФИ" Снежинский физикотехнический институт, Снежинск, Челябинская обл., Россия.

E-mail: n.r.sadykov@rambler.ru 
рассматривать НТ как бесконечно длинную электрическую цепь, единичный интервал которой характеризуется кинетической индуктивностью $L_{\mathrm{k}}$, квантовой емкостью $C_{\mathrm{q}}$, электростатической емкостью $C_{\text {eq }}$ и активным сопротивлением $R_{\mathrm{a}}$ на единицу длины [10]-[13], то углеродную НТ можно рассматривать как линию передачи, параметры которой определяются выражениями

$$
L_{\mathrm{k}}=\frac{h}{8 e^{2} v_{\mathrm{F}}}, \quad C_{\mathrm{q}}=\frac{8 e^{2}}{h v_{\mathrm{F}}}, \quad C_{\mathrm{eq}} \approx \frac{2 \pi}{\ln (a / 2 R)}, \quad R_{\mathrm{a}} \simeq \frac{h}{8 e^{2} v_{\mathrm{F}} \tau},
$$

где $a$ - расстояние до параллельно расположенной оси НТ плоскости, $e$ - заряд электрона, $h \approx 6.6 \cdot 10^{-34}$ Дж $\cdot \mathrm{c}-$ постоянная Планка, $e=1.6 \cdot 10^{-19}$ Кл - заряд электрона, $v_{\mathrm{F}}$ - скорость электронов в точке Ферми НТ. Суммарная электрическая емкость получается как последовательное соединение $C_{\mathrm{q}}$ и $C_{\mathrm{eq}}$. Такие уникальные свойства НТ приводят к уникальным эффектам при их взаимодействии с электромагнитным излучением или нестационарным электрическим полем [3], [10], [11], [14]-[20]; в частности, они позволяют рассмотреть эффект генерации СВЧ- и терагерцового излучения [21]-[23].

В настоящей работе исследована зависимость поверхностной плотности тока с учетом эффекта межоболочечного туннелирования в массиве невзаимодействующих друг с другом двухслойных углеродных нанотрубок (УНТ) при наличии нестационарного электрического импульса. В этом случае генерируются симметричная и асимметричная моды. Составляющая поверхностной плотности тока, соответствующая симметричной моде, может приводить к генерации СВЧ (или терагерцового) излучения. Показано, что для симметричной моды наличие массива двухслойных УНТ эквивалентно увеличению их концентрации в два раза в поперечном относительно оси НТ направлении по сравнению с массивом однослойных НТ, а также увеличению резонансной частоты в $\sqrt{2}$ раз. Показано, что при воздействии нестационарного электрического импульса на массив двухслойных УНТ за счет асимметричной моды для поверхностных токов также генерируется продольная электростатическая волна (несимметричная мода), которая не влияет на процесс генерации излучения. Полученные в работе результаты могут быть обобщены на случай многослойных НТ.

Рассматриваемая задача относится к формируемому в настоящее время новому направлению в науке о наноматериалах - наноэлектромагнетизму, суть которого состоит в объединении макроскопической электродинамики и микроскопической квантовой теории электронных свойств низкоразмерных структур, прежде всего НТ. При решении задачи наноэлектромагнетизма приходится использовать полуклассические методы и подходы. Например, для описания электронного транспорта в УНТ длиной $l \geqslant 1$ мкм используется полуклассический подход: скорость вычисляется в соответствии с каноническими уравнениями Гамильтона, но при этом гамильтониан определяется с помощью квантово-механического подхода. Среднее значение плотности тока вдоль оси $z$ однозначно выражается через функцию распределения носителей заряда, которая, в свою очередь, определяется из кинетического уравнения Больцмана в приближении времени релаксации и при пренебрежении эффектами, связанными с неоднородностью поля. Фигурирующая фермиевская равновесная фукция распределения также предполагает знание электронных уровней энергии. 
Другая ситуация возникает при $l \leqslant 1$ мкм. В этом случае для определения проводимости нужно воспользоваться формулой Ландауэра, соответствующей случаю баллистического транспорта. Квантово-механические расчеты затруднены из-за того, что число составляющих частиц велико. Применение классической теории сплошных сред не обоснованно из-за малого относительно числа атомов в составе НТ. Отсюда при рассмотрении НТ, кроме необходимости постоянно сравнивать теоретические и численные результаты с экспериментом, необходимы как привлечение, так и разработка новых теоретических методов.

Наноэлектромагнетизм является примером того, что количественное изменение масштабов задач приводит к качественным уникальным изменениям. Кроме таких понятий, как квантовое сопротивление, квантовая емкость, кинетическая индуктивность, в случае классического подхода при описании взаимодействия с УНТ или генерации излучения возникают новые эффекты, например существенное качественное отличие свойств макроскопических вибраторов от свойств наноантенн: ввиду большой кинетической индуктивности оптимальная длина наноантенн существенно меньше (на два порядка) длины волны излучения (определяется коэффициентом замедления). С учетом актуальности задачи генерации терагерцового и СВЧ-излучения на основе массива УНТ в поле мощных нестационарных электрических импульсов (она включает по сути три независимые области исследований) становится очевидной необходимость проведения теоретических исследований.

Приступим к рассмотрению сформулированной задачи.

\section{2. ЭЛЕКТРОДИНАМИКА ДВУХСЛОЙНОЙ НТ}

Большой интерес представляют массивы как однослойных, так и многослойных НТ. Например, в работе [24] проведен теоретический анализ распространения азимутально-симметричной направляемой волны (моды) в многослойной УНТ в инфракрасной области и в видимом диапазоне частот. Применительно к двухслойной УНТ исследовано влияние эффекта туннелирования между одностеночными УНТ и рассмотрено распространение излучения в волноводе из $N$ изолированных металлических УНТ конечной длины. Диаметр поперечного сечения пучка УНТ взят намного меньше длины волны излучения в вакууме. В работе [24] рассматривался случай сильного взаимодействия между УНТ, когда расстояние между НТ соизмеримо их диаметру. В работе [23] рассмотрен механизм воздействия нестационарного электрического поля на систему невзаимодействующих (практически уединенных) УНТ $(m, 0)$ типа “зигзаг” ( $m$ не кратно трем), авторы использовали уравнение Леонтовича-Левина (см. [25]), которое записано для нулевого члена плотности тока в разложении по малому параметру $X$. На основе полученных результатов исследована возможность генерации миллиметрового излучения. Результаты [23] обобщены в работе [26] на массив слабовзаимодействующих металлических УНТ (расстояние между НТ соизмеримо их длине) $(m, 0)$ типа "зигзаг” ( $m$ кратно трем). В статье [9] результаты работы [25] частично обобщаются на хиральные НТ.

Имеет место связь между электромагнитным 4-потенциалом $A^{i}=(\varphi, \mathbf{A})$ и вектором Герца электрического типа [27]

$$
\mathbf{B}=\operatorname{rot} \mathbf{A}, \quad \mathbf{A}=\frac{1}{c} \frac{\partial \vec{\Pi}}{\partial t}, \quad \varphi=-\vec{\nabla} \vec{\Pi},
$$


где $c$ - скорость света в вакууме, $\vec{\Pi}$ - вектор Герца, $(\varphi, \mathbf{A})$ - четырехмерный вектор-потенциал, В - вектор магнитной индукции. Предположим, что вектор $\vec{\Pi}=$ $\Pi_{z} \mathbf{e}_{z} e^{-i \omega t}$ параллелен оси НТ и является только функцией радиуса $\rho$, где $\omega-$ циклическая частота, $\mathbf{e}_{z}$ - направленный вдоль оси НТ единичный вектор. Тогда в соответствии с (2) для продольной компоненты электрического поля и азимутальной составляющей магнитного поля имеем

$$
\mathbf{B}=-i k \operatorname{rot} \vec{\Pi}, \quad B_{\varphi}=i k \frac{\partial \Pi_{z}}{\partial \rho}, \quad E_{z}=\left(\frac{\partial^{2}}{\partial z^{2}}+k^{2}\right) \Pi_{z},
$$

где $k=\omega / c$. С помощью тензора поверхностной проводимости $\sigma_{p q}$ по аналогии c [24], [28] получаем граничные условия на поверхности $R_{1}, R_{2}$ двухслойной НТ

$$
\begin{aligned}
&\left.B_{\varphi}\right|_{R_{1}+0}-\left.B_{\varphi}\right|_{R_{1}-0}=\frac{4 \pi}{c} \sigma_{11}\left[E_{1, z}^{(0)}+\left(\frac{\partial^{2}}{\partial z^{2}}+k^{2}\right)\left(\Pi_{1, z}\left(R_{1}, z\right)+\Pi_{2, z}\left(R_{1}, z\right)\right)\right]+ \\
&+\frac{4 \pi}{c} \sigma_{12}\left[E_{2, z}^{(0)}+\left(\frac{\partial^{2}}{\partial z^{2}}+k^{2}\right)\left(\Pi_{1, z}\left(R_{2}, z\right)+\Pi_{2, z}\left(R_{2}, z\right)\right)\right], \\
&\left.B_{\varphi}\right|_{R_{2}+0}-\left.B_{\varphi}\right|_{R_{2}-0}=\frac{4 \pi}{c} \sigma_{21}\left[E_{1, z}^{(0)}+\left(\frac{\partial^{2}}{\partial z^{2}}+k^{2}\right)\left(\Pi_{1, z}\left(R_{1}, z\right)+\Pi_{2, z}\left(R_{1}, z\right)\right)\right]+ \\
&+\frac{4 \pi}{c} \sigma_{22}\left[E_{2, z}^{(0)}+\left(\frac{\partial^{2}}{\partial z^{2}}+k^{2}\right)\left(\Pi_{1, z}\left(R_{2}, z\right)+\Pi_{2, z}\left(R_{2}, z\right)\right)\right],
\end{aligned}
$$

где $E_{1, z}^{(0)}, E_{2, z}^{(0)}-z$-компоненты падающего электрического поля $E_{z}^{(0)}$, которые действуют на одностенные УНТ с радиусами $R_{1}, R_{2}$.

Представим потенциал Герца в виде аксиально-симметричной волны:

$$
\begin{gathered}
\Pi_{z}=\Pi_{1, z}(\rho, z)+\Pi_{2, z}(\rho, z), \\
\Pi_{1, z}(\rho, z)=A_{1} \Psi_{1}(\rho) e^{i h_{0} z}, \quad \Pi_{2, z}(\rho, z)=A_{2} \Psi_{2}(\rho) e^{i h_{0} z} .
\end{gathered}
$$

Функцию $\Psi_{p}(\rho), p=1,2$, выберем в виде [28]

$$
\Psi_{p}(\rho)= \begin{cases}K_{0}\left(\kappa R_{p}\right) I_{0}(\kappa \rho), & \rho \leqslant R_{p} \\ I_{0}\left(\kappa R_{p}\right) K_{0}(\kappa \rho), & \rho>R_{p}\end{cases}
$$

где $\kappa^{2}=h_{0}^{2}-k^{2}$; принятый в [28] вид волновой функции отличается от волновой функции, взятой в работе [24], на константу $4 i \pi R_{p} / \omega$.

С учетом (3) и (6) левые части системы уравнений (4) преобразуем к виду

$$
\begin{aligned}
& \left.B_{\varphi}\right|_{R_{1} \pm 0}=i k\left\{\left.\left(\frac{\partial \Pi_{1, z}}{\partial \rho}\right)\right|_{R_{1} \pm 0}+\left.\left(\frac{\partial \Pi_{2, z}}{\partial \rho}\right)\right|_{R_{1} \pm 0}\right\} \\
& \left.B_{\varphi}\right|_{R_{2} \pm 0}=i k\left\{\left.\left(\frac{\partial \Pi_{1, z}}{\partial \rho}\right)\right|_{R_{2} \pm 0}+\left.\left(\frac{\partial \Pi_{2, z}}{\partial \rho}\right)\right|_{R_{2} \pm 0}\right\} .
\end{aligned}
$$

С учетом равенства

$$
K_{0}(x) \frac{d I_{0}(x)}{d x}-I_{0}(x) \frac{d K_{0}(x)}{d x}=\frac{1}{x}
$$


заменим левые части (4) в соответствии с равенствами (7):

$$
\begin{aligned}
\left.B_{\varphi}\right|_{R_{1}+0}-\left.B_{\varphi}\right|_{R_{1}-0}= & i k\left\{\left.\left(\frac{\partial \Pi_{1, z}}{\partial \rho}\right)\right|_{R_{1}+0}+\left.\left(\frac{\partial \Pi_{2, z}}{\partial \rho}\right)\right|_{R_{1}+0}\right\}- \\
& -i k\left\{\left.\left(\frac{\partial \Pi_{1, z}}{\partial \rho}\right)\right|_{R_{1}-0}+\left.\left(\frac{\partial \Pi_{2, z}}{\partial \rho}\right)\right|_{R_{1}-0}\right\}= \\
= & -i k A_{1} e^{i h_{0} z}=2 \frac{i k}{R_{1} X_{1}} \Pi_{1, z}\left(R_{1}, z\right), \\
\left.B_{\varphi}\right|_{R_{2}+0}-\left.B_{\varphi}\right|_{R_{2}-0}=i k\left\{\left.\left(\frac{\partial \Pi_{1, z}}{\partial \rho}\right)\right|_{R_{2}+0}+\left.\left(\frac{\partial \Pi_{2, z}}{\partial \rho}\right)\right|_{R_{2}+0}\right\}- & -i k\left\{\left.\left(\frac{\partial \Pi_{1, z}}{\partial \rho}\right)\right|_{R_{2}-0}+\left.\left(\frac{\partial \Pi_{2, z}}{\partial \rho}\right)\right|_{R_{2}-0}\right\}= \\
=- & i k A_{2} e^{i h_{0} z}=2 \frac{i k}{R_{2} X_{2}} \Pi_{2, z}\left(R_{2}, z\right),
\end{aligned}
$$

где по аналогии с [3], [4] введены обозначения

$$
X_{1}=-2 K_{0}\left(\kappa R_{1}\right) I_{0}\left(\kappa R_{1}\right), \quad X_{2}=-2 K_{0}\left(\kappa R_{2}\right) I_{0}\left(\kappa R_{2}\right)
$$

Из (4) с учетом (5) и (8) окончательно получаем уравнения для потенциала Герца

$$
\begin{aligned}
\frac{2 i k}{R_{1} X_{1}} \Pi_{11, z}=\frac{4 \pi}{c} & \sigma_{11}\left[E_{1, z}^{(0)}+\left(\frac{\partial^{2}}{\partial z^{2}}+k^{2}\right)\left(\Pi_{11, z}+\Pi_{12, z}\right)\right]+ \\
+ & \frac{4 \pi}{c} \sigma_{12}\left[E_{2, z}^{(0)}+\left(\frac{\partial^{2}}{\partial z^{2}}+k^{2}\right)\left(\Pi_{21, z}+\Pi_{22, z}\right)\right] \\
\frac{2 i k}{R_{2} X_{2}} \Pi_{22, z}=\frac{4 \pi}{c} & \sigma_{21}\left[E_{1, z}^{(0)}+\left(\frac{\partial^{2}}{\partial z^{2}}+k^{2}\right)\left(\Pi_{11, z}+\Pi_{12, z}\right)\right]+ \\
+ & \frac{4 \pi}{c} \sigma_{22}\left[E_{2, z}^{(0)}+\left(\frac{\partial^{2}}{\partial z^{2}}+k^{2}\right)\left(\Pi_{21, z}+\Pi_{22, z}\right)\right]
\end{aligned}
$$

где введены обозначения

$$
E_{1, z}^{(0)}=E_{z}^{(0)}\left(R_{1}, z\right), \quad E_{2, z}^{(0)}=E_{z}^{(0)}\left(R_{2}, z\right), \quad \widehat{\Pi}_{p q, z}=\left(\begin{array}{cc}
\Pi_{1, z}\left(R_{1}, z\right) & \Pi_{2, z}\left(R_{1}, z\right) \\
\Pi_{1, z}\left(R_{2}, z\right) & \Pi_{2, z}\left(R_{2}, z\right)
\end{array}\right)
$$

Введем новые функции

$$
\begin{aligned}
\widetilde{\Pi}_{z}(\rho, z) & =\Pi(\rho, z)+\Phi(\rho, z), & \widetilde{\Pi}_{z} & =\widetilde{\Pi}_{1, z}(\rho, z)+\widetilde{\Pi}_{2, z}(\rho, z), \\
\widetilde{\Pi}_{1, z} & =\widetilde{A}_{1} \Psi_{1}(\rho) e^{i h_{0} z}, & \widetilde{\Pi}_{2, z} & =\widetilde{A}_{2} \Psi_{2}(\rho) e^{i h_{0} z}, \\
E_{z}^{(0)} & =\left(\frac{\partial^{2}}{\partial z^{2}}+k^{2}\right) \Phi(\rho, z), & \Phi & =\Phi_{1}(\rho, z)+\Phi_{2}(\rho, z), \\
E_{1, z}^{(0)} & =\left(\frac{\partial^{2}}{\partial z^{2}}+k^{2}\right) \Phi_{1}\left(R_{1}\right), & E_{2, z}^{(0)} & =\left(\frac{\partial^{2}}{\partial z^{2}}+k^{2}\right) \Phi_{2}\left(R_{2}\right) .
\end{aligned}
$$


Из (9) и (10) следует система уравнений

$$
\begin{aligned}
& \left(\frac{\partial^{2}}{\partial z^{2}}+k^{2}\right) \sigma_{11}\left(\widetilde{\Pi}_{11, z}+\widetilde{\Pi}_{12, z}\right)+\left(\frac{\partial^{2}}{\partial z^{2}}+k^{2}\right) \sigma_{12}\left(\widetilde{\Pi}_{21, z}+\widetilde{\Pi}_{22, z}\right)- \\
& -\frac{i \omega}{2 \pi R_{1} X_{1}} \widetilde{\Pi}_{11, z}=-\frac{i \omega}{2 \pi R_{1} X_{1}} \Phi_{11}, \\
& \left(\frac{\partial^{2}}{\partial z^{2}}+k^{2}\right) \sigma_{21}\left(\widetilde{\Pi}_{11, z}+\widetilde{\Pi}_{12, z}\right)+\left(\frac{\partial^{2}}{\partial z^{2}}+k^{2}\right) \sigma_{22}\left(\widetilde{\Pi}_{21, z}+\widetilde{\Pi}_{22, z}\right)- \\
& -\frac{i \omega}{2 \pi R_{2} X_{2}} \widetilde{\Pi}_{22, z}=-\frac{i \omega}{2 \pi R_{2} X_{2}} \Phi_{22},
\end{aligned}
$$

где по аналогии с (5) введены величины

$$
\begin{aligned}
\widetilde{\Pi}_{p q, z} & =\Pi_{p q, z}+\Phi_{p q, z}, \\
\widetilde{\Pi}_{p q, z} & =\left(\begin{array}{ll}
\widetilde{\Pi}_{1, z}\left(R_{1}, z\right) & \widetilde{\Pi}_{2, z}\left(R_{1}, z\right) \\
\widetilde{\Pi}_{1, z}\left(R_{2}, z\right) & \widetilde{\Pi}_{2, z}\left(R_{2}, z\right)
\end{array}\right), \\
\Phi_{p q, z}\left(R_{1}, z\right) & =\left(\begin{array}{ll}
\Phi_{1, z}\left(R_{1}, z\right) & \Phi_{2, z}\left(R_{1}, z\right) \\
\Phi_{1, z}\left(R_{2}, z\right) & \Phi_{2, z}\left(R_{2}, z\right)
\end{array}\right) .
\end{aligned}
$$

Матрицы $\widetilde{\Pi}_{p q, z}$ из (12) можно записать в виде

$$
\widetilde{\Pi}_{p q, z}=\sum_{f=1}^{2} M_{p f} A_{f q} e^{i h_{0} z}=(\widehat{M} \widehat{A})_{p q} e^{i h_{0} z},
$$

где $\widehat{M}=M_{p q}$ и $\widehat{A}=A_{p q}$ имеют вид

$$
\begin{gathered}
M_{p q}=\left(\begin{array}{ll}
\Psi_{1}\left(R_{1}\right) & \Psi_{2}\left(R_{1}\right) \\
\Psi_{1}\left(R_{2}\right) & \Psi_{2}\left(R_{2}\right)
\end{array}\right)=\left(\begin{array}{cc}
K_{0}\left(\kappa R_{1}\right) I_{0}\left(\kappa R_{1}\right) & K_{0}\left(\kappa R_{2}\right) I_{0}\left(\kappa R_{1}\right) \\
K_{0}\left(\kappa R_{2}\right) I_{0}\left(\kappa R_{1}\right) & K_{0}\left(\kappa R_{2}\right) I_{0}\left(\kappa R_{2}\right)
\end{array}\right), \\
\widehat{A}=A_{p q}=\left(\begin{array}{cc}
\widetilde{A}_{1} & 0 \\
0 & \widetilde{A}_{2}
\end{array}\right) .
\end{gathered}
$$

Из (11) с учетом (13) и (14) получаем

$$
\begin{aligned}
& \left\{\left(\frac{\partial^{2}}{\partial z^{2}}+k^{2}\right)\left[(\hat{\sigma} \widehat{M})_{11} \widetilde{A}_{1}+(\hat{\sigma} \widehat{M})_{12} \widetilde{A}_{2}\right]-i \frac{\omega}{2 \pi R_{1} X_{1}} M_{11} \widetilde{A}_{1}\right\} e^{i h_{0} z}=-i \frac{\omega}{2 \pi R_{1} X_{1}} \Phi_{11}, \\
& \left\{\left(\frac{\partial^{2}}{\partial z^{2}}+k^{2}\right)\left[(\hat{\sigma} \widehat{M})_{21} \widetilde{A}_{1}+(\hat{\sigma} \widehat{M})_{22} \widetilde{A}_{2}\right]-i \frac{\omega}{2 \pi R_{2} X_{2}} M_{22} \widetilde{A}_{2}\right\} e^{i h_{0} z}= \\
& =-i \frac{\omega}{2 \pi R_{2} X_{2}} \Phi_{22} .
\end{aligned}
$$

Из (15) следует система однородных уравнений относительно $\widetilde{A}_{p}$. Нетривиальное решение этой системы уравнений дает дисперсионное уравнение для постоянной распространения $h_{0}=h_{0}(\xi)[24]$

$$
\operatorname{det}(\widehat{G} \cdot \widehat{M}-\widehat{B})=0,
$$

где $\widehat{G}=\sigma_{p q}, \widehat{M}=M_{p q}, \widehat{B}=i \omega \delta_{q p} / 4 \pi \kappa^{2} R_{p}$. 


\section{3. ЭЛЕКТРОДИНАМИКА ДВУХСЛОЙНОЙ НТ ПРИ ВОЗДЕЙСТВИИ НЕСТАЦИОНАРНОГО ЭЛЕКТРИЧЕСКОГО ИМПУЛЬСА}

Из полученных в работе [24] результатов следует, что априорная величина - частота межоболочечного туннелирования - имеет порядок $\omega_{\mathrm{t}}=10^{13} \mathrm{paд} / \mathrm{c}$. В [24] показано, что везде, за исключением окрестности $\omega_{t}$, туннелирование слабо влияет на симметричную моду и сильно влияет на асимметричную моду для поверхностных токов в широком диапазоне частоты. В окрестности $\omega_{\mathrm{t}}$ при наличии туннелирования для симметричной моды коэффициент усиления порядка единицы, а асимметричная мода имеет такое же значение для коэффициента замедления, что и симметричная мода в отсутствие межоболочечного туннелирования $\omega \leqslant 2 \omega_{\mathrm{t}}$. В настоящей работе нас интересует резонансная частота однослойных УНТ $\omega \cong 2 \cdot 10^{12} \mathrm{c}^{-1}$. В этом случае $\left(\omega \ll 2 \omega_{\mathrm{t}}\right)$ за счет туннельного эффекта реализуется только асимметричная мода. Поскольку нас интересует симметричная мода при частотах $\omega \ll 2 \omega_{\mathrm{t}}$, то нужно пренебречь туннельным эффектом, что, в свою очередь, приводит к установлению соотношения [24]

$$
\sigma_{q p}=\frac{\sigma_{0} \delta_{q p}}{R_{p}}
$$

где $\delta_{q p}$ - дельта-функция Кронекера. Тогда в соответствии с [3], [4] будет иметь место соотношение

$$
\sigma_{0} \approx \frac{4 i e^{2} v_{\mathrm{F}}}{\pi h(\omega+i \nu)}=\frac{2 i v_{\mathrm{F}}}{\pi R_{0}(\omega+i \nu)}
$$

где $R_{0}=h / 2 e^{2}$ - обратное значение кванта проводимости (кванта сопротивления).

Из (16) и (17) следует

$$
(\hat{\sigma} \widehat{M})_{q p}=\frac{\sigma_{0} M_{q p}}{R_{q}} .
$$

С учетом (18) систему уравнений (15) запишем по аналогии с [23], [26], [29] в виде дифференциальных уравнений (для этого воздействуем на (15) оператором $\partial^{2} / \partial z^{2}+$ $\left.k^{2}\right)$

$$
\begin{aligned}
& {\left[\frac{\partial^{2}}{\partial z^{2}}+\omega^{2}\left(\frac{1}{c^{2}}+\frac{\pi}{4 \alpha c v_{\mathrm{F}}\left|X_{1}\right|}\right)+i \frac{\pi}{4 \alpha c v_{\mathrm{F}}\left|X_{1}\right|} \nu \omega\right] M_{11} \widetilde{A}_{1}+\left(\frac{\partial^{2}}{\partial z^{2}}+k^{2}\right) M_{12} \widetilde{A}_{2}=} \\
& \quad=\frac{i \omega}{2 \pi \kappa^{2} X_{1} \sigma_{0}} E_{1, z}^{(0)} e^{-i h_{0} z}, \\
& \left(\frac{\partial^{2}}{\partial z^{2}}+k^{2}\right) M_{21} \widetilde{A}_{1}+\left[\frac{\partial^{2}}{\partial z^{2}}+\omega^{2}\left(\frac{1}{c^{2}}+\frac{\pi}{4 \alpha c v_{\mathrm{F}}\left|X_{2}\right|}\right)+i \frac{\pi}{4 \alpha c v_{\mathrm{F}}\left|X_{2}\right|} \nu \omega\right] M_{22} \widetilde{A}_{2}= \\
& \quad=\frac{i \omega}{2 \pi \kappa^{2} X_{2} \sigma_{0}} E_{2, z}^{(0)} e^{-i h_{0} z} .
\end{aligned}
$$

При выводе (19) учтено, что имеют место равенства (см. (10))

$$
\begin{gathered}
\left(\frac{\partial^{2}}{\partial z^{2}}+k^{2}\right) \widetilde{A}_{\alpha} e^{i h_{0} z}=-\kappa^{2} \widetilde{A}_{\alpha} e^{i h_{0} z} \\
E_{1, z}^{(0)}=\left(\frac{\partial^{2}}{\partial z^{2}}+k^{2}\right) \Phi_{1}\left(R_{1}\right), \quad E_{2, z}^{(0)}=\left(\frac{\partial^{2}}{\partial z^{2}}+k^{2}\right) \Phi_{2}\left(R_{2}\right) .
\end{gathered}
$$


Из системы дисперсионных уравнений (19) следует система дифференциальных уравнений

$$
\begin{aligned}
& \frac{\partial^{2} \widetilde{A}_{1}}{\partial t^{2}}+\nu \frac{\partial \widetilde{A}_{1}}{\partial t}+\omega_{1}^{2} \widetilde{A}_{1}+\omega_{1}^{2} \frac{M_{12}}{M_{11}} \widetilde{A}_{2}=\frac{\beta_{1}^{2} c^{2}}{2 \pi \kappa^{2} \sigma_{0} X_{1} M_{11}} \frac{\partial E_{1, z}^{(0)}}{\partial t} e^{-i h_{0} z} \\
& \omega_{2}^{2} \frac{M_{21}}{M_{22}} \widetilde{A}_{1}+\frac{\partial^{2} \widetilde{A}_{2}}{\partial t^{2}}+\nu \frac{\partial \widetilde{A}_{2}}{\partial t}+\omega_{2}^{2} \widetilde{A}_{2}=\frac{\beta_{2}^{2} c^{2}}{2 \pi \kappa^{2} \sigma_{0} X_{2} M_{22}} \frac{\partial E_{2, z}^{(0)}}{\partial t} e^{-i h_{0} z},
\end{aligned}
$$

где

$$
\begin{array}{rlrl}
\beta_{1}^{2}=\left[1+\frac{c}{v_{\mathrm{F}}} \frac{\pi}{4 \alpha X_{1}}\right]^{-1}, & \beta_{2}^{2} & =\left[1+\frac{c}{v_{\mathrm{F}}} \frac{\pi}{4 \alpha X_{2}}\right]^{-1}, \\
\omega_{1}^{2}=\beta_{1}^{2} \kappa^{2} c^{2}, & \omega_{2}^{2}=\beta_{2}^{2} \kappa^{2} c^{2}
\end{array}
$$

Из $(21)$ в случае однослойных УНТ $\left(E_{2, z}^{(0)}=0, M_{21}=0, \widetilde{A}_{2}=0\right)$ следует уравнение для поверхностной плотности тока. Действительно, для одномерного случая с учетом результатов [25] имеем

$$
j_{z}\left(R_{1}, z\right)=\frac{\sigma_{0}}{R_{1}}\left(\frac{\partial^{2}}{\partial z^{2}}+k^{2}\right) \widetilde{\Pi}_{1, z}=-\frac{\kappa^{2} \sigma_{0}}{R_{1}} \widetilde{A}_{1} M_{11} e^{i h_{0} z}
$$

где $\sigma_{0}$ из $(17)$ имеет размерность $[\Omega]$.

С учетом (23) из (21) получаем уравнение для поверхностного тока для уединенной УНТ при воздействии нестационарного электрического импульса [30]:

$$
\frac{\partial^{2} j_{z}}{\partial t^{2}}+\nu \frac{\partial j_{z}}{\partial t}+\omega_{1}^{2} j_{z}=-\frac{\beta_{1}^{2} c^{2}}{2 \pi R_{1} X_{1}} \frac{\partial E_{1, z}^{(0)}}{\partial t} e^{-i h_{0} z},
$$

которое совпадает с приведенным в работе [26] аналогичным выражением для массива УНТ в пренебрежении взаимным влиянием отдельных УНТ.

При $R_{2}-R_{1} \approx R_{1}, \nu \ll \sqrt{\omega_{1} \omega_{2}}$ из $(21)$ следует выражение для резонансной частоты

$$
\omega_{\mathrm{r}}^{2}=\frac{\omega_{1}^{2}+\omega_{2}^{2}}{2} \pm \frac{1}{2}\left[\left(\omega_{1}^{2}-\omega_{2}^{2}\right)^{2}+4 \omega_{1}^{2} \omega_{2}^{2} \frac{M_{12} M_{21}}{M_{11} M_{22}}\right]^{1 / 2} .
$$

Система уравнений (21) позволяет определить амплитуды $\widetilde{A}_{1}$ и $\widetilde{A}_{2}$ для функций $\widetilde{\Pi}_{1, z}$ и $\widetilde{\Pi}_{2, z}$ из $(10)$. Зная эти функции, можно вычислить плотность тока на поверхности однослойных УНТ в соответствии со следующей формулой:

$$
j_{p, z}^{(0)}\left(R_{p}, z\right)=-\kappa^{2} \sum_{p=1}^{2}(\hat{\sigma} \widehat{M})_{q p} \widetilde{A}_{p} e^{i h_{0} z}
$$

В случае НТ для оптимальной длины антенны $l$ (см. с. 434 в монографии [11] или работу [12]) имеет место соотношение $l=\lambda \operatorname{Re} \beta_{1} / 2$. Поэтому по аналогии с [23], [26], [29] положим

$$
\lambda \approx 1 \mathrm{MM}, \quad l=\frac{\lambda \operatorname{Re} \beta_{1}}{2} \approx 10 \mathrm{MKM}, \quad R_{1}=3 \mathrm{HM}, \quad R_{2}=2 R_{1} .
$$


Тогда получаем $\kappa R_{1} \approx h_{0} R_{1}=2 \pi R_{1} / \lambda \operatorname{Re} \beta_{1}=\pi R_{1} / l \approx 10^{-3}, \kappa R_{2} \approx 2 \cdot 10^{-3}$. Из (25) следует

$$
\omega_{\mathrm{r}}^{2}=\frac{\omega_{1}^{2}+\omega_{2}^{2}}{2} \pm \frac{1}{2}\left[\left(\omega_{1}^{2}-\omega_{2}^{2}\right)^{2}+4 \omega_{1}^{2} \omega_{2}^{2} \frac{K_{0}\left(\kappa R_{2}\right)}{K_{0}\left(\kappa R_{1}\right)} \frac{I_{0}\left(\kappa R_{2}\right)}{I_{0}\left(\kappa R_{1}\right)}\right]^{1 / 2}
$$

где при выводе учтено, что

$$
\begin{gathered}
\frac{M_{12} M_{21}}{M_{11} M_{22}}=\frac{K_{0}\left(\kappa R_{2}\right)}{K_{0}\left(\kappa R_{1}\right)} \frac{I_{0}\left(\kappa R_{2}\right)}{I_{0}\left(\kappa R_{1}\right)}, \\
K_{0}(\alpha \ll 1) \approx-0.577-\ln \frac{\alpha}{2}, \quad I_{0}(\alpha \ll 1) \approx 1+\frac{\alpha^{2}}{4} .
\end{gathered}
$$

Из (22) следует

$$
\left(\frac{\omega_{1}}{\omega_{2}}\right)^{2}=\left(\frac{\beta_{1}}{\beta_{2}}\right)^{2} \approx \frac{X_{1}}{X_{2}}=\frac{K_{0}\left(\kappa R_{1}\right) I_{0}\left(\kappa R_{1}\right)}{K_{0}\left(\kappa R_{2}\right) I_{0}\left(\kappa R_{2}\right)} \approx 1.1
$$

В результате с учетом (29) высокочастотное решение (27) запишем в виде (симметрично-подобная мода [24])

$$
\omega_{\mathrm{r}}^{(1)} \approx\left(2 \omega_{0}^{2}\right)^{1 / 2}, \quad \omega_{0}^{2}=\frac{\omega_{1}^{2}+\omega_{2}^{2}}{2}, \quad \widetilde{A}_{1} \approx \widetilde{A}_{2} .
$$

Решение (30) позволяет получить поверхностные высокочастотные продольные токи.

Второе (низкочастотное) решение имеет вид (асимметрично-подобная мода [24])

$$
\omega_{\mathrm{r}}^{(2)} \approx\left(0.047 \omega_{0}^{2}\right)^{1 / 2} \approx 0.22 \omega_{0}, \quad \widetilde{A}_{1} \approx-\widetilde{A}_{2}
$$

Решение (31) позволяет получить поверхностные низкокочастотные продольные токи.

Из (30) следует, что поверхностные продольные токи, которые соответствуют симметрично-подобной моде, распространяются вдоль однослойных НТ с одинаковой фазой $\left(\widetilde{A}_{1} \approx \widetilde{A}_{2}\right)$, причем резонансная частота увеличивается примерно в $\sqrt{2}$ раз по сравнению со случаем, когда рассматривается однослойная НТ [23], [29]. Таким образом, в этом случае массив слабовзаимодействующих двухслойных НТ с точки зрения задачи генерации излучения [23], [26], [29] приводит к увеличению концентрации НТ и уменьшению величины задержки $\left\{\beta_{\mathrm{r}}^{(1)}\right\}^{-1} \approx h_{0} / \omega_{\mathrm{r}}^{(1)}$ (к увеличению коэффициента замедления $\left.\beta_{\mathrm{r}}^{(1)}\right)$.

Из (31) следует, что в случае асимметричной моды поверхностные продольные токи распространяются вдоль однослойных НТ в противофазе $\left(\widetilde{A}_{1} \approx-\widetilde{A}_{2}\right)$, причем резонансная частота уменьшается на порядок по сравнению со случаем, когда рассматривается однослойная НТ. В этом случае возникает качественно новый эффект - генерация продольного электромагнитного поля. Это поле не является полем излучения, но оно позволяет определить поверхностные продольные токи. Такое поле в классической электродинамике можно получить между вставленными друг в друга соленоидами, оси которых совпадают: токи текут в противоположных направлениях, но совпадают по абсолютной величине; количества витков на 
единицу длины двух соленоидов совпадают. При этом следует подчеркнуть, что возбудить такую асимметричную моду сложно. С помощью нестационарных мощных электрических импульсов волн это практически невозможно сделать [30]. Такая асимметричная мода по своим свойствам подобна продольной электростатической волне [24], локализованной между однослойными НТ. Продольная электростатическая волна реализуется при учете туннельного эффекта, но в отсутствие магнитного поля и, как следствие, в отсутствие продольных поверхностных токов (в этом случае реализуется ситуация $\left.\sigma_{11} \sigma_{22}=\sigma_{12} \sigma_{21}\right)$.

Из (22) следует, что величина задержки в случае симметричной моды (30) равняется $\left\{\beta_{\mathrm{r}}^{(1)}\right\}^{-1} \approx h_{0} / \omega_{\mathrm{r}}^{(1)}$, а величина задержки асимметричной моды (31) равняется $\left\{\beta_{\mathrm{r}}^{(2)}\right\}^{-1} \approx h_{0} / \omega_{\mathrm{r}}^{(2)}$. Отношение величин задержки асимметричной и симметричной мод составляет $\left\{\beta_{\mathrm{r}}^{(2)}\right\}^{-1} /\left\{\beta_{\mathrm{r}}^{(1)}\right\}^{-1}=\omega_{\mathrm{r}}^{(1)} / \omega_{r}^{(2)} \approx 7$. Аналогичное значение получено в работе [24] в отсутствие туннелирования.

\section{4. ВЛИЯНИЕ ИЗГИБА ПОВЕРХНОСТЕЙ НА ЭЛЕКТРОДИНАМИКУ ДВУХСЛОЙНОЙ НТ}

В УНТ большого радиуса с металлическим или полупроводниковым типом проводимости вклад в электропроводность дают несколько зон, в то время как в металлических хиральных УНТ $(n, m)$, где $n \neq m$, с малым радиусом изогнутый слой индуцирует большую энергетическую щель. При рассмотрении транспортных свойств НТ мы пренебрегали тем, что они представляют собою изогнутую поверхность, т. е. тем, что транспортные свойства, в частности проводимость, должны зависеть от радиуса НТ. Рассмотрим этот вопрос применительно к нашей задаче более подробно.

Изгиб поверхности нарушает двумерную изотропность электронных свойств УНТ [31], [32]. В этом случае интеграл перекрытия для $\pi$-электронов зависит от направления касательной к изогнутой поверхности. Как следствие, для металлических УНТ $(n, m)$, где $n \neq m$, появляется узкая (в терагерцовом диапазоне) запрещенная зона, что, в свою очередь, начинает влиять на электропроводность отдельной УНТ [9]. Изгиб НТ позволяет скорректировать кинетическую индуктивность и приводит к возникновению в эквивалентной электрической цепи дополнительной $R L C$-ветви, связанной с терагерцовым квантовым переходом. Дополнительный терагерцовый переход может качественно модифицировать электронный транспорт и проводимость УНТ, которые не являются идеальными проводниками. Хотя энергетическая щель лежит в терагерцовом диапазоне, на практике может представлять интерес диапазон 100 ГГц, поскольку за счет изгиба ширина линии перехода может быть большой [33], [34]. Поскольку при рассмотрении задачи взаимодействия нестационарных импульсов [30] нас интересует этот диапазон частот, рассмотрим влияние изогнутой поверхности на транспортные свойства НТ.

Используя формализм, изложенный в работе [35], запишем аксиальную проводимость в виде

$$
\begin{aligned}
\tilde{\kappa} & =-2 \int_{E_{\mathrm{gc}}}^{\infty} \sqrt{1-\left(\frac{E_{\mathrm{gc}}}{E}\right)^{2}}\left(\frac{d F}{d E}\right) d E, \quad \tilde{\kappa}_{1}=F\left(-E_{\mathrm{gc}}\right)-F\left(E_{\mathrm{gc}}\right), \\
\sigma_{z z} & =\frac{4 v_{\mathrm{F}}}{R_{0} 2 \pi R}\left[\tilde{\kappa} \frac{1}{-i \omega+\nu}+\tilde{\kappa}_{1} \frac{\omega}{i\left(\omega^{2}-\omega_{0}^{2}\right)+\nu_{1} \omega}\right],
\end{aligned}
$$


где $E(k) \cong \pm \sqrt{E_{\mathrm{gc}}^{2}+\hbar^{2} v_{\mathrm{F}}^{2} k^{2}}, E_{\mathrm{gc}}=\gamma a_{0}^{2} / 32 R^{2}$ - ширина запрещенной зоны, определяемая кривизной изогнутой поверхности УНT, $R$ - радиус $\mathrm{HT}, \omega_{0}=2 E_{\mathrm{gc}} / \hbar-$ частота квантового перехода, обусловленного изгибом поверхности УНТ. Резонансная частота $\omega_{0}$ относится к низкочастотной границе зоны $\Omega_{\mathrm{ot}}$, в которой в межзонном переходе нельзя пренебречь соотношением $\omega_{0} \cong 0.07 \Omega_{\mathrm{ot}}\left(a_{0} / R\right)$. Таким образом,

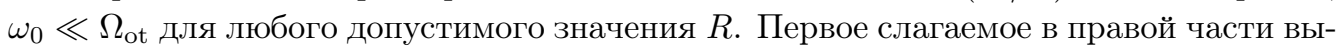
ражения для $\sigma_{z z}(32)$ соответствует пределу $\tilde{\kappa} \rightarrow 1$, т. е. $E_{\mathrm{gc}} \rightarrow 0$. Второе слагаемое в правой части выражения (32) связано с низкочастотным резонансом $\pi$-электронов на квазидискретном уровне, где должно выполняться условие $\omega_{0} \gg \nu_{1}$. Для УНТ $(n, 0)$ типа "зигзаг" с большим значением $R$ имеем $\tilde{\kappa} \cong 1, \tilde{\kappa}_{1} \cong 0(n>15, R>0.6$ нм $)$. Для малого радиуса $\tilde{\kappa} \cong 0, \tilde{\kappa}_{1} \cong 1$.

Покажем, что в интересующем нас диапазоне $\lambda \approx 1 \mathrm{мm}\left(\omega=2 \pi c / \lambda \approx 1.9 \cdot 10^{12} \mathrm{c}^{-1}\right)$ второе слагаемое в квадратных скобках в (32) значительно меньше первого слагаемого. Для УНТ $(n, 0)$ типа "Зигзаг" при $n=15$ имеет место $\omega_{0}=2 E_{\mathrm{gc}} / \hbar=$ $\pi^{2} \gamma / 4 n^{2} \hbar \approx 4.6 \cdot 10^{13} \mathrm{c}^{-1}$. Таким образом, справедливо $\omega / \omega_{0} \approx 0.04 \ll 1$. Поскольку в соответствии с результатами работы [9] при $n=15$ имеет место $\tilde{\kappa} \approx \tilde{\kappa}_{1} \approx 1 / 2$, то в интересующем нас диапазоне частот второе слагаемое в квадратных скобках в (32) можно преобразовать к виду

$$
\tilde{\kappa}_{1} \frac{\omega}{i\left(\omega^{2}-\omega_{0}^{2}\right)+\nu \omega} \approx i \frac{\tilde{\kappa}_{1}}{\omega_{0}^{2}}\left(1-i \frac{\nu_{1}}{\omega_{0}^{2}} \omega\right) \omega .
$$

С учетом (33) проводимость из (32) примет вид

$$
\sigma_{z z} \approx i \frac{2 v_{\mathrm{F}}}{\pi R R_{0}}\left[\tilde{\kappa} \frac{1}{\omega+i \nu}+\frac{\tilde{\kappa}_{1}}{\omega_{0}^{2}}\left(1-i \frac{\nu_{1}}{\omega_{0}^{2}} \omega\right) \omega\right],
$$

откуда следует

$$
\sigma_{z z} \approx i \frac{2 v_{\mathrm{F}}}{\pi R R_{0}(\omega+i \nu)}\left(\tilde{\kappa}+\frac{\omega^{2}}{\omega_{0}^{2}} \tilde{\kappa}_{1}\right) .
$$

Из $(16),(17)$ и $(35)$ видно, что поскольку при $\omega / \omega_{0} \approx 0.04, n=15$ имеет место $\left(\tilde{\kappa}+\tilde{\kappa}_{1} \omega^{2} / \omega_{0}^{2}\right) \approx \tilde{\kappa} \approx 1 / 2$, то проводимость уменьшается примерно в два раза. Это означает, что в интересующем нас диапазоне частот можно пренебречь квантовыми переходами между валентной зоной и зоной проводимости.

\section{5. ВОЗМОЖНОСТЬ ГЕНЕРАЦИИ СВЧ- И ТЕРАГЕРЦОВОГО ИЗЛУЧЕНИЯ С ПОМОЩЬЮ МАССИВА ДВУХСЛОЙНЫХ УНТ}

Полученные в настоящей работе результаты позволяют обобщить задачу генерации СВЧ-излучения [23], [26], [29] с помощью нестационарного импульса большой мощности в среде [30] на основе массива невзаимодействующих однослойных УНТ. Для этого получим сначала систему уравнений для поверхностных токов на стенках двухслойной УНТ. Из (3) и (10) в отсутствие туннельного эффекта следуют выражения для поверхностных токов

$$
\begin{aligned}
& j_{z, 1}=j_{z}\left(R_{1}, t\right)=-\frac{\kappa^{2} \sigma_{0}}{R_{1}}\left(\widetilde{\Pi}_{11, z}+\widetilde{\Pi}_{12, z}\right), \\
& j_{z, 2}=j_{z}\left(R_{2}, t\right)=-\frac{\kappa^{2} \sigma_{0}}{R_{2}}\left(\widetilde{\Pi}_{21, z}+\widetilde{\Pi}_{22, z}\right) .
\end{aligned}
$$


С учетом (36) из (21) получаем систему уравнений для поверхностных токов

$$
\begin{aligned}
& \frac{\partial^{2} j_{z, 1}}{\partial t^{2}}+\nu \frac{\partial j_{z, 1}}{\partial t}+\omega_{1}^{2} j_{z, 1}+\omega_{2}^{2} \frac{M_{12}}{M_{22}} j_{z, 2}= \\
& =-\frac{c^{2}}{2 \pi R_{1}}\left\{\frac{\beta_{1}^{2}}{X_{1}} \frac{\partial E_{1, z}^{(0)}}{\partial t}+\frac{\beta_{2}^{2}}{X_{2}} \frac{M_{12}}{M_{22}} \frac{\partial E_{2, z}^{(0)}}{\partial t}\right\} e^{-i h_{0} z} \\
& \frac{\partial^{2} j_{z, 2}}{\partial t^{2}}+\nu \frac{\partial j_{z, 2}}{\partial t}+\omega_{2}^{2} j_{z, 1}+\omega_{1}^{2} \frac{M_{21}}{M_{22}} j_{z, 2}= \\
& =-\frac{c^{2}}{2 \pi R_{2}}\left\{\frac{\beta_{2}^{2}}{X_{2}} \frac{\partial E_{2, z}^{(0)}}{\partial t}+\frac{\beta_{1}^{2}}{X_{1}} \frac{M_{21}}{M_{11}} \frac{\partial E_{1, z}^{(0)}}{\partial t}\right\} e^{-i h_{0} z}
\end{aligned}
$$

С помощью системы уравнений (37) можно осуществить переход к случаю однослойной НТ (24).

Для изучения случая генерации СВЧ-излучения по аналогии с [23], [26], [29] нужно перейти к поверхностной плотности токов

$$
J_{z, 1}=N j_{z, 1}, \quad J_{z, 2}=N j_{z, 2},
$$

где $N$ - концентрация НТ в поперечном к массиву УНТ направлении. Уравнение поля излучения имеет вид

$$
\frac{\partial^{2} E(t, x)}{\partial t^{2}}-v_{1}^{2} \frac{\partial^{2} E(t, z)}{\partial x^{2}}=-\frac{1}{\varepsilon_{0}}\left(\frac{v_{1}}{c}\right)^{2} \frac{\partial J(t, x)}{\partial t},
$$

где зависимость плотности тока от продольной координаты и времени определяется профилем мощного нестационарного импульса $E_{1, z}^{(0)} \approx E_{2, z}^{(0)} \sim f\left(t-x / v_{1}\right)$.

\section{6. ЗАКЛЮЧЕНИЕ}

Полученная для двухслойных УНТ система уравнений $(21)$ для амплитуд $\widetilde{A}_{1}$ и $\widetilde{A}_{2}$ функций $\widetilde{\Pi}_{1, z}$ и $\widetilde{\Pi}_{2, z}(10)$ позволяет численно определить плотность тока на поверхности однослойных УНТ в соответствии с формулой (26). В случае двухслойных УНТ длины $l \approx 10$ мкм (длина волны излучения $\lambda \sim 1 \mathrm{мм}$ ) с радиусами $R_{1}=3$ нм, $R_{2}=2 R_{1}$ в отсутствие туннельного эффекта для симметричной моды $\left(\widetilde{A}_{1} \approx \widetilde{A}_{2}\right)$ частота собственных колебаний $\omega_{\mathrm{r}}^{(1)} \approx\left(2 \omega_{0}\right)^{1 / 2} \approx 1.9 \cdot 10^{12} \mathrm{c}^{-1}$, а для асимметричной моды $\left(\widetilde{A}_{1} \approx-\widetilde{A}_{2}\right)$ частота собственных колебаний $\omega_{\mathrm{r}}^{(2)} \approx 0.22 \omega_{0} \approx 4.2 \cdot 10^{11} \mathrm{c}^{-1}$. Резонансная частота для симметричной моды увеличивается примерно в $\sqrt{2}$ раз по сравнению со случаем однослойной НТ, а в случае асимметричной моды - уменьшается на порядок. Для СВЧ-излучения с длиной волны $\lambda \approx 1$ мм можно пренебречь изгибом УНТ, который приводит к квантовому терагерцовому переходу с частотой $\omega_{0} \gg \omega$. Полученная плотность тока на поверхности однослойных УНТ позволяет рассмотреть задачу генерации СВЧ-излучения в массиве двухслойных УНТ по аналогии с массивом однослойных слабовзаимодействующих УНТ [23], [26], [29]. Кроме увеличения резонансной частоты примерно в $\sqrt{2}$ раз, для массива невзаимодействующих двухслойных НТ мы получили увеличение концентрации НТ при неизменном расстоянии между соседними НТ. 


\section{Список литературы}

[1] O. M. Yevtushenko, G. Ya. Slepyan, S. A. Maksimenko, A. Lakhtakia, D. A. Romanov, Phys.Rev. Lett., 79:6 (1997), 1102-1105.

[2] G. Ya. Slepyan, S. A. Maksimenko, A. Lakhtakia, O. M. Yevtushenko, Phys.Rev. B, 57:16 (1998), 9485-9497.

[3] G. Ya. Slepyan, S. A. Maksimenko, A. Lakhtakia, O. M. Yevtushenko, A. V. Gusakov, Phys. Rev. B, 60:24 (1999), 17136-17149.

[4] C. А. Максименко, Г. Я. Слепян, Радиотехника и электроника, 47:3 (2002), 261-280.

[5] С.А. Максименко, Г.Я. Слепян, Вестник фонда фундаментальных исследований, 2006, № 4, 92-113.

[6] S. A. Maksimenko, G. Ya. Slepyan, "Nano electromagnetics of low-dimensonal structures", The Handbook of Nanotechnology: Nanometer structure Theory, Modeling, and Simulation, ed. A. Lakhtakia, SPIE Press, Bellingham, 2004, 145-206.

[7] S. A. Maksimenko, G. Ya. Slepyan, K. B. Batrakov, A. A. Khrushchinsky, P. P. Kuznir, A. M. Nemilentsau, M. V. Shuba, "Electromagnetic waves in carbon nanostructures", Carbon Nanotubes and Related Structures E, eds. V. Blank, B. Kulnitskiy, Research Signpost Publisher, Trivandrum, India, 2008, 147-187.

[8] M. J. Hagmann, IEEE Trans. on Nanotechnol., 4:2 (2005), 289-296.

[9] G. Miano, C. Forestiere, A. Maffucci, S. A. Maksimenko, G. Ya. Slepyan, IEEE Trans. on Nanotechnol., 10:1 (2011), 135-149.

[10] C. Forestiere, A. Maffucci, S. A. Maksimenko, G. Miano, G. Ya. Slepyan, IEEE Trans. on Nanotechnol., 11:3 (2012), 554-564.

[11] П. Н. Дьячков, Электронные свойства и применение нанотрубок, БИНОМ. Лаборатория знаний, М., 2011.

[12] C. Rutherglen, P. Burke, Small, 5:8 (2009), 884-906.

[13] P. Burke, S. Li, Z. Yu, IEEE Trans. on Nanotechnol., 5:4 (2006), 314-334.

[14] О.В. Кибис, М.Е. Портной, Писъма в ЖТФ, 31:15 (2005), 85-89.

[15] K. G. Batrakov, S. A. Maksimenko, P. P. Kuzhir, C. Thomsen, Phys.Rev. B., 79:12 (2009), 125408, $12 \mathrm{pp}$.

[16] G. Ya. Slepyan, M. V. Shuba, S. A. Maksimenko, C. Thomsen, A. Lakhtakia, Phys. Rev. B, 81:20 (2010), 205423, 6 pp.

[17] K. G. Batrakov, O. V. Kibis, P. P. Kuzhir, M. Rosenau da Costa, M. E. Portnoi, J. Nanophoton., 4:1 (2010), 041665, 29 pp.

[18] O. V. Kibis, M. Rosenau da Costa, M. E. Portnoi, Nano Lett., 7:11 (2007), 3414-3417.

[19] M. E. Portnoi, O. V. Kibis, M. Rosenau da Costa, Superlattices and Microstructures, 43:5-6 (2008), 399-407.

[20] М. Б. Белоненко, С. Ю. Глазов, Н. Е. Мещеряков, ФТП, 44:9 (2010), 1248-1253.

[21] Н. Р. Садыков, Н. А. Скоркин, ФТП, 46:2 (2012), 168-173.

[22] Н. Р. Садыков, Н. А. Скоркин, ФТП, 46:6 (2012), 809-814.

[23] Н. Р. Садыков, Н. А. Скоркин, Оптика атмосферы и океана, 25:4 (2012), 344-348.

[24] M. V. Shuba, G. Ya. Slepyan, S. A. Maksimenko, C. Thomson, A. Lakhtakia, Phys. Rev. B, 79:15 (2009), 155403, 17 pp.

[25] G. Ya. Slepyan, M. V. Shuba, S. A. Maksimenko, A. Lakhtakia, Phys. Rev. B, 73:19 (2006), 195416, $11 \mathrm{pp}$.

[26] Н. Р. Садыков, Н. А. Скоркин, Оптика атмосферы и океана, 26:2 (2013), 160-165.

[27] В. В. Батыгин, И.Н. Топтыгин, Современная электродинамика. Часть 1. Микроскопическая теория, ИКИ, М.-Ижевск, 2003.

[28] M. V. Shuba, S. A. Maksimenko, A. Lakhtakia, Phys. Rev. B, 76:24 (2007), 155407, 9 pp.

[29] Н. Р. Садыков, Н. А. Скоркин, ФТП, 46:8 (2012), 1043-1048. 
[30] Г. А. Месяц, М. И. Яландин, УФН, 175:3 (2005), 225-246.

[31] C. I. Kane, E. J. Mele, Phys. Rev. Lett., 78:10 (1997), 1932-1935.

[32] M. F. Lin, Phys. Rev. B, 58:7 (1998), 3629-3632.

[33] T. Kampfrath, K. von Volkmann, C. M. Aguirre, P. Desjardins, R. Martel, M. Krenz, C. Frischkorn, M. Wolf, L. Perfetti, Phys. Rev. Lett., 101:26 (2008), 267403, 4 pp.

[34] F. Borondics, K. Kamarás, M. Nikolou, D. B. Tanner, Z. H. Chen, A. G. Rinzler, Phys. Rev. B., 74:4 (2006), 045431, 6 pp., arXiv: cond-mat/0512667.

[35] S. A. Maksimenko, G. Ya. Slepyan, "Electrodynamic properties of carbon nanotubes", Electromagnetic Fields in Unconventional Materials and Structures, eds. O.N. Singh, A. Lakhtakia, Wiley, New York, 2000, 217-255.

Поступила в редакцию 27.02.2013, после доработки 16.04.2013 